\title{
Estudo da efetividade de um programa de triagem auditiva neonatal universal
}

\section{Effectiveness study of the universal newborn hearing screening}

Juliana Cristina Fernandes ${ }^{1}$

M árcia Regina N ozawa ${ }^{2}$

${ }^{1}$ Departamento de Enfermagem, Unicamp. Rua Tessália Vieira de Camargo 126, Caixa Postal 6111, Cidade Universitária Zeferino Vaz. 13083-887 Campinas SP. jc.fer@valvin.net

${ }^{2}$ Departamento de Enfermagem, Faculdade de Ciências M édicas, Universidade Estadual de Campinas.
Abstract This article discusses the insufficiency of family participation in a program of $U$ niversal Newborn Hearing Screening. As it is a premature detection of hearing alterations, with the possibility of reduction of damages caused by these, it becomes essential the family participation during all the diagnosis process. The aim of thetext is to understand the absence of the mothers of infants born between M ay 2002 and June 2004 at Center of Integral Attention to the $\mathrm{H}$ ealth of the Woman (Unicamp) when returning to a second hearing evaluation. Through the characterization of social-demographic profiles of the mothers, some aspects were related to the non-returning at the screener service and three mothers were selected to interview. It was concluded that three traces from the mother's profiles are detached among mothers who returned less times: to have more than one child; to have frequency of only one to three prenatal consultations; and not having a partner. The basic allegation for the nonreturning was that the loss of hearing could be identified through observation at home. Besides being constituted by social factors, the absences of the mothers reflect cultural practices. It was considered necessary the creation of a net of health services that guide the mothers of infants about alterations in the infantile development.

Key words Child health, Family adhesion, H earing loss
Resumo Este artigo discute a insuficiência de participação familiar num programa de triagem auditiva neonatal universal. Por se tratar da detecção precoce de alterações auditivas, com a possibilidade de redução dos prejuízos causados por estas, torna-se fundamental a partici pação familiar durante todo o processo de diagnóstico e reabilitação. 0 objetivo do texto é compreender a ausência das mães dos lactentes nascidos entre maio de 2002 ejunho de 2004 no Centro de Atenção Integral à Saúde da M ulher (CAISM -U nicamp) ao retorno para a segunda avaliação auditiva. Através da caracterização do perfil sociodemográfico das mães, algunsaspectos foram relacionados ao não retorno ao serviço de triagem etrês mães selecionadas para entrevista. Concluiu-se que três traços do perfil materno se destacam dentre as que menos retornaram ao serviço: possuir mais de um filho; ter frequência de apenas uma a três consultas pré natais; e não possuir companheiro. A principal alegação para o não retorno foi de que as perdas auditivas seriam passíveis de identificação pela observação em casa. Assim, além de ser constituída por fatores sociais, a ausência das mães reflete práticas culturais. Considera-se necessária a criação de uma rede de serviços de saúde que oriente gestantes e mães de lactentes sobre as alterações no desenvolvimento infantil. Palavras-chave Saúdeinfantil, Adesão familiar, Perda auditiva 
Introdução

0 presente artigo aborda o tema da detecção precoce da surdez no Brasil, enfocando, especificamente, a não adesão de mães de lactentes a programas de triagem auditiva neonatal universal (TANU), sobre o qual há escassa bibliografia no país. Pretende-se com este estudo apresentar contribuições ao campo da avaliação de programas de saúde infantil e ao aprimoramento de serviços que favoreçam a qualidade de vida da população.

A tual mente, a surdez éconsiderada como um problema de saúde pública em virtude de sua significativa prevalência, mas, sobretudo, pelas múltiplas consequências que pode acarretar ao desenvolvimento humano nos aspectos intelectuais, sociais, linguísticos, cognitivos, emocionais eculturai $s^{1-4}$. A incapacidade de detectar e perce ber os sons, em qualquer grau, prejudica o processo de aquisição da fala e o desenvolvimento da linguagem, necessários para uma melhor integração de indivíduos nas sociedades, cuja forma de comunicação é, predominantemente, oral ${ }^{5}$.

Ao observarmos dados do Censo Brasileiro de 2000 sobre a incidência das distintas deficiências, constatamos uma estimativa de cerca de $24,5 \mathrm{mi}$ Ihões de pessoas ( $14,5 \%$ da população) portadoras de al gum tipo de incapacidade para enxergar, ouvir, mover-se ou de al guma deficiência física e mental ${ }^{6}$. Dentre elas, a deficiência auditiva éa tercerra maisfrequenteno país, representando $16,7 \%$ das ocorrências, aproximando-seàs estimativas de países que adotam a mesma conceituação nas pesquisas demográficas, tais como Estados Unidos (15\%) eAustrália (18\%), por exemplo6.

Entre os recém-nascidos, as alterações auditivas ocorrem em, aproximadamente, um a três de cada 1.000 neonatos considerados com baixo risco para a surdez, e em 2 a $4 \%$ de recém-nascidos provenientes das unidades de terapia intensiva (UTI) ${ }^{7}$. No entanto, $50 \%$ das perdas auditivas são identificadas em crianças consideradas de baixo risco para a surdez $z^{8-10}$.

Diante dessa realidade, significativas contribuições foram sendo apresentadas no esforço de reduzir os prejuízos causados pela surdez. Estes estudos, especialmente os de Yoshinaga-Itano et al. ${ }^{11,12}$, indicam que, quando as alterações auditivas são diagnosticadas até os três meses de idade e a intervenção terapêutica é iniciada até os seis meses, o desenvolvimento da compreensão e da expressão da linguagem, bem como o relacionamento social e desempenho acadêmico da criança com alterações auditivas, pode ser compatível com o de crianças ouvintes da mesma faixa etária. Assim, amplia-se a triagem auditiva neonatal universal (TANU) definida como um processo de avaliação da audição infantil que permitea detecção de possíveis alterações auditivas, abrangendo todos os lactentes, incluindo os considerados livres de indicadores de risco para surdez, recomendando a implantação desses programas nas maternidade ${ }^{10}$.

Neste sentido, em 1971, formaliza-se, nos Estados Unidos, através do Joint Committe on Infant $\mathrm{H}$ earing $(\mathrm{JClH})$, a primeira recomendação para a implantação da triagem auditiva neonatal nas maternidades. Tal comitê representa 0 principal órgão de recomendação da triagem, reconhecido no mundo todo, inclusiveno Brasil.

No Brasil, a primeira iniciativa de disseminação de informações e implantação da TANU foi desencadeada pelo Grupo de Apoio a Triagem Auditiva N eonatal Universal (GATANU) em 1998. No ano seguinte, divulga-se a primeira recomendação nacional para sua realização, elaborada pelo Comitê Brasileiro sobre Perdas Auditivas na Infância (CBPAI) ${ }^{7}$. Atualmente, alguns municípios do Estado de São Paulo possuem legislação própria no que tange ao oferecimento do procedimento de avaliação auditiva nas maternidades.

De acordo com o $\mathrm{JCIH}^{10}$, um programa efetivo deTANU deve, em seus seis primeiros meses de implantação, triar, no mínimo, 95\% de lactentes e reavaliar, pelo menos, $70 \%$ dos lactentes reencaminhados. Superado esse período, o programa deverá obter $95 \%$ de retorno dos lactentes encaminhados para o reteste após a primeira fase da triagem.

Para tanto, a efetividade de um programa de TANU, no que tange à detecção e ao diagnóstico precoce de alterações auditivas e à pronta inserção das crianças em programas específicos de reabilitação, dependeda disponibilidade do equipamento adequado, de profissionais habilitados para a realização dos testes, da oferta do serviço nas maternidades, mas, sobretudo, do envolvimento e participação dosfamiliares durantetodo o processo.

Para que isso ocorra, é necessário que haja a participação familiar nesseprocesso tanto paraque a criança seja levada atéo local onde serão realizados os testes, como para que, nos casos de detecção de perda auditiva, a família possa ser orientada e integrada aos programas de reabilitação.

Contudo, o envolvimento familiar éconsiderado insatisfatório em muitos programas nacionais e internacionais, afetando, negativamente, as metas a serem alcançadas ${ }^{13}$. 
No Estado de São Paulo, estudos de avaliação de serviços de triagem auditiva no primeiro ano de funcionamento indicam os seguintes percentuais de não retorno à conclusão da triagem: em Itanhaém, 30\% ${ }^{14}$; na Maternidade do Hospital Universitário de São Paulo, 26,9\% 15 ; e no Centro de Estudos e Pesquisas em Reabilitação Professor Gabriel Porto da Universidade Estadual de Campinas, $47,7 \%$ e $28,6 \%{ }^{16}$. Estudos norte-americanos $^{17}$ apontam índices de não retorno à segunda etapa da avaliação auditiva de lactentes submetidos à primeira triagem auditiva na maternidade de $32,5 \%$ no Texas, e de $48,9 \%$, em Nova York ${ }^{18}$. Por sua vez, em uma pesquisa européia, encontrou-se o retorno de apenas 7,7\% ${ }^{19}$. Esses índices são considerados insatisfatórios em referência às metas que garantiriam a qualidade do programa detriagem auditiva, definidas pelo $\mathrm{JCIH}$.

Verifica-se que, em geral, os programas de triagem auditiva atingem a primeira meta de qualidade proposta9,16,20; no entanto, 0 alcance das metas de $70 \%$ de retornos nos primeiros seis meses de implantação e de $95 \%$ após o primeiro ano de funcionamento constitui-se em um desafio permanente.

Diante dessas considerações, esta pesquisa teve por objetivo compreender a ausência de retorno das mães dos lactentes nascidos no período de maio de 2002 a junho de2004, no Centro deAtenção Integral à Saúde da Mulher (CAISM ), da Universidade Estadual de Campinas (Unicamp), à segunda avaliação auditiva no programa de TANU, desenvolvido pelo Centro de Estudos e Pesquisas em Reabilitação Professor Gabriel O. S. Porto (CEPRE). Para isso, buscou-se, por um lado, caracterizar o perfil demográfico e social das mães assistidas no período assinalado, para as quais foi indicado o retorno para segunda avaliação auditiva de seus filhos; por outro, comparar o perfil materno de acordo com o comparecimento ou não à segunda avaliação e, por fim, apreender os motivos al egados por algumas mães para o não comparecimento ao serviço.

Esta pesquisa foi financiada durante vinte meses pelo Consel ho $\mathrm{N}$ acional deD esenvolvimento Científico e Tecnológico (CNPq), através do Programa de Bolsas de M estrado no País.

\section{Método}

Projeto de pesquisa aprovado pelo Comitê de Ética em Pesquisa da Faculdade de Ciências Médicas (FCM ), Unicamp, através de Parecer no 082/ 2005 de 24 de maio de 2005.
Adotou-se o modelo de estudo descritivo ${ }^{21} \mathrm{e}$ circunscreveu-se ao programa de TANU desenvolvido pelo CEPRE da Faculdade de Ciências M édicas (FCM) da Unicamp. Constituem-se sujeitos deste estudo mães dos lactentes que nasceram no CAISM, no período de maio de 2002 a junho de 2004, permaneceram no alojamento conjunto, foram submetidos aos testes da triagem auditiva e que, pelos resultados inconclusivos ou negativos, necessitariam retornar ao CEPRE para uma segunda avaliação. As mães dos lactentes que permaneceram na unidadede terapia intensiva neonatal foram excluídas do estudo, pois esses foram submetidos a procedimentos específicos de avaliação auditiva, realizada por equipe e tecnologia distintas.

Para a caracterização do perfil materno, inicialmente, procedeu-se à sistematização de dados secundários extraídos do banco de dados do Sistema de Informações sobre $\mathrm{N}$ ascidos Vivos (SINASC) do município de Campinas.

Foram registrados no banco de dados sobre os nascidos vivos (SINASC) deste município, aproximadamente, 27.817 nascimentos no município, dentre os quais, 6.231 (22,4\%) ocorridos no CAISM, no período em questão. As variáveis estudadas corresponderam à faixa etária, escolaridade, ocupação, estado civil, número de filhos, frequência ao pré natal e região de residência da família, de acordo com a área de abrangência dos centros de saúdes do município. Os dados foram transportados do software Windows Excel e processados com o uso do software Epi Info 2002.

Paralelamente, buscou-se, nos arquivos do CEPRE, os registros dos lactentes que realizaram a triagem auditiva na maternidade e necessitariam ser, novamente, avaliados ( $\mathrm{N}=770)$. Esses dados, confrontados com o banco do SINASC, permitiram a identificação das respectivas variáveis de caracterização materna. N a sequência, tal população foi subdividida em dois subgrupos: mães de lactentes conduzidos (451), correspondendo a $58,6 \%$ do total, e mães de lactentes não conduzidos à segunda avaliação auditiva (319), representando $41,4 \%$ do conjunto total (770). No tocante ao tratamento estatístico, utilizou-se o teste de qui-quadrado ou exato de Fisher (para valores esperados menores que 5) ${ }^{22}$, para a análise da associação entre duas variáveis categóricas, o teste de M ann-W hitney ${ }^{23,24}$ na análise comparativa das variáveis numéricas entre os dois grupos. Na relação entre as variáveis e o não retorno à triagem, foi utilizada a análise de regressão logística, modelo logito ${ }^{25}$ e, ainda, efetuadas as análises univariada e multivariada com crité- 
rio Stepwise de seleção de variáveis. 0 nível de significância adotado para os testes estatísticos foi de $5 \%(p<0.05)$.

Após a análise estatística comparativa entre os dois subgrupos, identificou-se que a distinção significativa entre eles ocorria nas variáveis: número de filhos, frequência a consultas pré-natais e estado civil. Tal perfil configurou os parâmetros utilizados para a escolha de três mães que não retornaram para a segunda avaliação auditiva, com as quais se buscou contato direto, por meio de entrevistas, a fim de apreender os sentidos do não comparecimento ao serviço, como expressão da realidade construída por esses sujeitos sociais ${ }^{26}$. As entrevistas foram gravadas e realizadas com base em um roteiro de questões abertas com 0 intuito de promover a livre expressão das mães ${ }^{27}$.

0 referencial teórico para a reflexão sobre os elementos relacionados com o não comparecimento das famílias aos programas de TANU fundamentou-se no acesso à saúde como categoria analítica. Para tanto, tomou-se por base o modelo comportamental indicado por Andersen ${ }^{28}$, que prioriza as características do indivíduo inserido na comunidade e a organização do sistema de saúde, englobando a entrada aos serviços de saúde e a continuidade do tratamento. Para este autor, o uso de serviços de saúde é mediado por fatores predisponentes dos usuários (idade, escolaridade, condição social, etc.), por fatores capacitantes (comunidadeefamília) e pelas necessidades de saúde, percebidas e avaliadas, que interferem na busca eutilização dos serviços de saúde.

Os fatores capacitantes corresponderiam às características externas ao indivíduo que exercem influência em suas decisões sobre sua saúde, tais como a família e a comunidade na qual se insere. A necessi dade percebida se traduz na maneira como as pessoas entendem sua saúde, como experienciam os sintomas da doença, dor, como julgam o problema etomam iniciativa de procurar um profissional de saúde. E, ainda, a necessidade avaliada, a qual corresponderia àquela determinada e resultante do avanço científico da medicina e do treinamento e competência dos profissionais. A análise da necessidade percebida auxilia a compreensão da procura ena aderência ao sistema de cuidados, enquanto a necessidade avaliada possibilita a análise do tipo e quantidade de tratamento provido depois que o usuário se apresenta ao serviço de saúde.

Esta proposta de análise lança mão de ele mentos do modelo de crenças para explicar o uso dos serviços, especialmente o comportamento preventivo de saúde, uma vez que dele decorre a procura espontânea da população. N estes casos, a percepção do indivíduo sobre sua necessidade de saúde é considerada como uma variável não mutável; no entanto, a imediata razão para a utilização dos serviços de saúde é que pode ser alterada por intermédio de programas de educação e conscientização de saúde. Similarmente, a necessidade avaliada também teria o potencial de influenciar a utilização dos serviços de saúde, uma vez quepodealterar o juízo ou a opinião dos indivíduos em relação aos cuidados de saúde.

\section{Resultados e discussão}

A identificação do perfil das mães quenecessitariam conduzir seus filhos à segunda triagem auditiva permitiu verificar as seguintes características predominantes. No que diz respeito à idade materna, $67,1 \%$ do grupo total situa-se na faixa etária de 20 e 34 anos de idade, com uma participação de $66,3 \%$ entre as mães que retornaram e $68,3 \%$ entre as que não retornaram para a conclusão da triagem. Já a distribuição das mães segundo a ocupação indicou que, do grupo total, $79,4 \%$, não exerciam atividade remunerada, percentual que variava de $77 \%$ entre as mães que retornaram e $82,8 \%$ que não retornaram.

Em relação ao município de residência, $63,6 \%$ das famílias são do próprio município de Campinas. A distribuição das famílias de acordo com a região de moradia em Campinas (Tabela 1) não indica diferencial significativo em relação ao retorno ou não retorno à conclusão da triagem, mesmo para o distrito de saúde norte, no qual o programa de triagem estudado se insere. Nesse sentido, a proximidade ou distância física entreo serviço e a moradia das famílias, ou o acesso geográfico, não as distinguiu no comportamento de retorno à segunda triagem.

H ouve diferença estatisticamente significativa entre os dois subgrupos de mães em relação às variáveis: estado civil materno, o número de fiIhos e a frequência ao pré-natal. A Tabela 2 revela que $62 \%$ de mães com companheiro retornaram para a segunda aval iação auditiva, enquanto apenas $54,5 \%$ das mães sem companheiro o fizeram. Assim, a probabilidade de não retorno das mães que viviam sem companheiro foi 1,4 vezes maior em relação àquelas que vivem acompanhadas.

$\mathrm{Na}$ Tabela 3, observa-se que, quanto maior $\mathrm{O}$ número defilhos, menor éa frequência de retorno das mães. Mulheres com mais de um filho apresentam a probabilidade 1,5 vezes maior de 
não retornar ao serviço em relação às mães primigestas, tendência que se amplia para as mães com dois filhos e para aquelas com três filhos ou mais (com probabilidade de não retorno 2,0 e 2,7 vezes maior, respectivamente). A Tabela 4, por sua vez, ilustra que quanto menor éa frequência das mães às consultas pré-natais, menor também é o retorno para a segunda avaliação auditiva. A probabilidade de não retorno das mães que realizaram de uma a três consultas prénatais é 3,1 vezes maior em relação àquelas quefrequentaram a quatro ou mais consultas.
Destaca-se também a relevância da escolaridade materna em relação aos percentuais de retorno para a conclusão da triagem auditiva. O bserva-se, na Tabela 5, que o aumento da escolaridade materna refletido em percentuais mais elevados de retorno à segunda avaliação, dado particularmente acentuado para aquelas de escolaridade igual ou maior a doze anos e, inversamente, nos percentuais mais reduzidos para as mães com os menores níveis de escolaridade.

Associada ao perfil materno, as alegações para o não retorno à segunda avaliação auditiva per-

Tabela 1. Distribuição das mães, segundo o retorno e o não retorno à segunda avaliação auditiva e a região de moradia. Campinas, 2006.

\begin{tabular}{|c|c|c|c|c|c|c|}
\hline \multirow{2}{*}{ Região de moradia } & \multicolumn{2}{|c|}{ Retornaram } & \multicolumn{2}{|c|}{$\mathrm{N}$ ão retornaram } & \multicolumn{2}{|c|}{ Total subgrupo } \\
\hline & $\mathrm{n}$ & $\%$ & $\mathrm{n}$ & $\%$ & $n$ & $\%$ \\
\hline Norte & 127 & 57,2 & 95 & 42,8 & 222 & 100,0 \\
\hline Noroeste & 17 & 65,4 & 9 & 34,6 & 63 & 100,0 \\
\hline Leste & 75 & 61,5 & 47 & 38,5 & 122 & 100,0 \\
\hline Sudoeste & 30 & 55,6 & 24 & 44,4 & 26 & 100,0 \\
\hline Sul & 37 & 58,7 & 26 & 41,3 & 54 & 100,0 \\
\hline
\end{tabular}

Fonte: SINASC, 2004.

Tabela 2. Distribuição das mães, segundo o retorno e o não retorno à segunda avaliação auditiva e o estado civil. Campinas, 2006.

\begin{tabular}{|c|c|c|c|c|c|c|}
\hline \multirow{2}{*}{ Estado civil } & \multicolumn{2}{|c|}{ Retorno } & \multicolumn{2}{|c|}{ Não retorno } & \multicolumn{2}{|c|}{ Total subgrupo } \\
\hline & $n$ & $\%$ & $n$ & $\%$ & $n$ & $\%$ \\
\hline Com companheiro & 258 & 62,0 & 158 & 38,0 & 416 & 100,0 \\
\hline Sem companheiro & 188 & 54,5 & 157 & 45,5 & 345 & 100,0 \\
\hline
\end{tabular}

Fonte: SIN ASC, 2004.

Tabela 3. Distribuição das mães, segundo o retorno ou não retorno à segunda avaliação auditiva e o número de filhos. Campinas, 2006.

\begin{tabular}{|c|c|c|c|c|c|c|}
\hline \multirow{2}{*}{ Número de filhos } & \multicolumn{2}{|c|}{ Retorno } & \multicolumn{2}{|c|}{ Não retorno } & \multicolumn{2}{|c|}{ Total } \\
\hline & $n$ & $\%$ & $\mathrm{n}$ & $\%$ & $\mathrm{n}$ & $\%$ \\
\hline Primigesta & 220 & 67,5 & 106 & 32,5 & 326 & 100,0 \\
\hline 1 filho & 121 & 58,7 & 85 & 41,3 & 206 & 100,0 \\
\hline 2 filhos & 60 & 50,4 & 59 & 49,6 & 119 & 100,0 \\
\hline 3 ou mais filhos & 50 & 42,0 & 69 & 58,0 & 119 & 100,0 \\
\hline
\end{tabular}

IC 95\% para a OR para não retorno.

Fonte: SINASC, 2004. 
Tabela 4. Distribuição das mães, segundo o retorno ou não retorno à segunda avaliação auditiva e a frequência ao pré-natal. Campinas, 2006.

\begin{tabular}{|c|c|c|c|c|c|c|}
\hline \multirow{2}{*}{ Frequência } & \multicolumn{2}{|c|}{ Retorno } & \multicolumn{2}{|c|}{ Não retorno } & \multicolumn{2}{|c|}{ Total } \\
\hline & $\mathrm{n}$ & $\%$ & $\mathrm{n}$ & $\%$ & $\mathrm{n}$ & $\%$ \\
\hline 1 consulta & 2 & 20,0 & 8 & 80,0 & 10 & 100,0 \\
\hline 2 consultas & 20 & 39,2 & 31 & 60,8 & 51 & 100,0 \\
\hline 3 consultas & 98 & 52,4 & 89 & 47,6 & 187 & 100,0 \\
\hline 4 consultas & 317 & 63,9 & 179 & 36,1 & 496 & 100,0 \\
\hline 5 ou mais consultas & 14 & 53,8 & 12 & 46,2 & 26 & 100,0 \\
\hline
\end{tabular}

IC 95\% para a OR para não retorno. Fonte: SINASC, 2004.

Tabela 5. Distribuição das mães, segundo o retorno e o não retorno à segunda avaliação auditiva e a escolaridade. Campinas, 2006.

\begin{tabular}{|c|c|c|c|c|c|c|}
\hline \multirow{2}{*}{ Escolaridade } & \multicolumn{2}{|c|}{ Retorno } & \multicolumn{2}{|c|}{ Não retorno } & \multicolumn{2}{|c|}{ Total subgrupo } \\
\hline & $n$ & $\%$ & $\mathrm{n}$ & $\%$ & $n$ & $\%$ \\
\hline 0 - 3 anos de estudo & 6 & 37,5 & 10 & 62,5 & 16 & 100,0 \\
\hline 4 - 7 anos de estudo & 244 & 58,1 & 176 & 41,9 & 420 & 100,0 \\
\hline 8 - 11 anos de estudo & 157 & 57,0 & 118 & 43,0 & 275 & 100,0 \\
\hline$\geq 12$ anos de estudo & 39 & 73,6 & 14 & 26,4 & 53 & 100,0 \\
\hline
\end{tabular}

Fonte: SINASC, 2004.

mitiram compor um quadro do conjunto de fatores predisponentes do usuário relativos aos obstáculos no acesso ao programa de TANU. Estes se relacionam tanto à organização do programa de TANU oferecido, cuja restrição de horário de atendimento e insuficiência de informações acerca da importância da triagem afetam o acesso dos usuários, quanto à carência de recursos materiais para deslocamento até o serviço, à necessidade de conduzir todos os filhos ao local de retorno, muitas vezes, pela ausência de retaguarda familiar ou social, ou ainda, à incompreensão materna acerca da necessidade de retornar para a segunda avaliação auditiva e o desconhecimento da tecnologia especificamente utilizada para esse fim, o que interfere na capacidade de reconhecimento das necessidades de saú de de seus filhos.

0 enunciado abaixo descrito exemplifica a convicção de uma mãe, que apesar de ter acompanhado a execução da triagem auditiva de seu filho no período do pós-parto imediato, acredi- ta que o exame otoscópico, realizado nas consultas de puericultura, permitiria identificar déficits auditivos.

Todas consultas que eu levei el e no posto, quea pediatra examinou, nunca teve problema no ouvido. Se a médica que examina fala que tem alguma coisa com o aparelhinho que elas olham, com certeza vão falar alguma coisa ( $M$ ãe A).

Na mesma direção, há outros indícios que reiteram a influência da necessidade avaliada (informada pelo profissional de saúde), no tipo de contato estabelecido pelas usuárias com os serviços de saúde. 0 julgamento que o profissional realiza sobre a condição de saúde da criança examinada, constitui-se, pois, num elemento primordial. Os depoimentos abaixo ilustram a posição de saber quase "absoluto", atribuída pelas mães aos médicos. Assim, na ausência de uma clara indicação do profissional para o retorno da criança à segunda avaliação auditiva, tal procedimento é por elas entendido como desnecessário. 
Eu não recebi [ papel do encaminhamento para o reteste] porque, se eu tivesse recebido, com certeza, o médico que me deu os papéis no dia da alta, tinha falado. Eles não falaram nada e eu vi só o papel do teste do pezinho. Setem que voltar, com certeza eles avisam, eles tem que explicar, né? ( $M$ ãe A).

Porque, assim, sobre isso, eles não me passaram nada, também não me deram papel nenhum. Ela faz acompanhamento com a pediatra dela [no centro de saúde] ea pediatra dela tá sempreol hando enão falou nada [a respeito da triagem auditiva] ( $M$ ãe $F$ ).

Em síntese, a crença das mães na capacidade dos médicos de avaliar necessidades acerca de medidas ou procedimentos voltados à promoção e proteção da saúde das crianças apresentou-se como um dos componentes à não adesão familiar ao programa.

A despeito do trabalho da equipe de triagem em prover informações às mães acerca da necessidade de repetição do teste, há depoimentos que também indicam a insuficiência desse processo deesclarecimento.

Se tivesse me falado, eu estava lembrando porque eu lembro de todos os papéis e de tudo que falaram pra mim (M ãe A).

Chegaram a falar do teste da orelhinha, mas não me falaram nada para voltar ( $M$ ãe F).

Associado a esses fatores há ainda a percepção das mães de que a observação empírica das reações de seus filhos às falas, sons e ruídos em geral, atestariam um desenvolvimento auditivo saudável. Como ilustrado pelos enunciados que se seguem abaixo, tal percepção também se relaciona ao não retorno ao serviço de triagem.

Bater palma para ele assi $\mathrm{m}$, 0 chocalho eu balançava para ele, barulho alto mesmo, sabe? Balançava a chave. Chamava ele. A primeira vez que chamou ele [pelo nome próprio], ele já atendia, nossa, o menino tá ótimo! ( $M$ ãe A).

Eu observo assim, por causa que ela fala muito bem, ela é uma criança muito esperta mesmo! Às vezes, ela fala baixo no meu ouvido. Brincar com as outras crianças também, ela brinca muito bem (Mãe F).

Atéhoje ele nunca, nem dor de ouvido ele teve, nada! O uvesuper bem, chama ele, eleatendesuper bem, nunca teve problema. Ele ouve! N ossa, ele é sapeca, nossa! Ele parece um foguete. Passa algum barulho, bato palma assim, ele reage ao barulho, então, por isso que eu não fiquei preocupada. Porque, com certeza, se ele tivesse com o ouvido tampado alguma coisa na hora que eu chamasse ele, batessepalma, elenão ia atender, então, eleatende super bem ( $M$ ãe $A)$.
A [criança] vai fazer três anos e ela é uma criança, assim, de surpreender. Ela passa assim por uma criança de cinco. Ela é bem mais ágil que a minha filha mais velha ( $M$ ãe $F$ ).

De fato, as maneiras pelas quais as mães observam a capacidade auditiva de seus filhos não se apresentam como inadequados para tal fim. No entanto, nos casos de perda auditiva leve ou moderada, o diagnóstico ocorre tardiamente, na mai oria das vezes, na idade escolar. Neste caso, a mãe ou outro cuidador da criança, e mesmo 0 serviço básico de saúde, não teriam a capacidade de diagnosticar alterações estruturais e orgânicas relativas à audição $0^{29}$. Além disso, a presença de dor ou desconforto nem sempre se associa à surdez, dois fatores que comumente aceleram a busca pelos serviços de saúde. Este aspecto da observação materna sobre a capacidade de audição dos filhos indica uma prática social arraigada e bastante associada ao não retorno, uma vez que permeia a vida cotidiana das pessoas e transcende, muitas vezes, a capacidade de interferência de profissionais ou serviços de saúde.

E ainda esse processo, quando associado ao desconhecimento dos prejuízos causados pelos déficits auditivosao desenvolvimento intelectual, social, linguístico, cognitivo eemocional das pessoas, pode resultar em resistências à adesão aos programas preventivos de saúde ${ }^{30}$.

\section{Conclusão}

A análise do perfil sociodemográfico materno apresenta, num primeiro momento, uma semeIhança em relação às características demográficas esociais encontradas em outras pesqui sas ${ }^{13,16}$. N o entanto, nesta investigação, a análise comparativa entre os dois subgrupos evidencia diferenças estatisticamente significativas entre ambos.

Retomando-se os pontos centrais, é possível afirmar que a faixa etária, a ocupação e a localização da residência em relação ao serviço desaúde se mostraram pouco relevantes em relação ao retorno ou ao não retorno para a segunda avaliação auditiva. Em contrapartida, destacam-setrês características maternas fortemente associadas ao não retorno à segunda avaliação auditiva, com grande relevância estatística: a baixa frequência às consultas pré-natais (de uma a três consultas); a presença de mais de um filho na família; e a ausência de companheiro.

Considera-se relevante, ainda, no conjunto dessas características, os dados referentes à escolaridade materna no sentido de que, quanto me- 
nor é o número de anos de estudos de uma mãe, menor também será a probabilidade de sua participação ao retorno.

Além da carência econômica das famílias eda falta de retaguarda de apoio social, que limitam a condição de adesão ao programa, a restrição no horário de atendimento foram indicados pelas mães como obstáculos ao acesso ao serviço de triagem. De modo semelhante, as estratégias educativas ou metodologias pedagógicas utilizadas na educação em saúde parecem não ter favorecido a incorporação de significados à triagem auditiva neonatal e, portanto, não afetaram as necessidades percebidas pelas mães.

Dessa forma, a falta de audição não se constitui, para parcela significativa das mães, em um problema que necessitaria de preocupação ou de investigação junto a profissionais especializados. Parece-Ihes suficiente a sua capacidade, pelos meios empíricos de avaliação, de constatar quea criança ouve bem.

Sugere-se que abordagem acerca da importância da audição no desenvolvimento da criança e a possibilidade concreta de detecção precoce de perdas auditivas seja adotada, especialmente, nas consultas pré natais, na tentativa de introduzir novos elementos para reflexão materna acerca da surdez infantil e seus prejuízos. Esta ação de saúde poderia ampliar a possibilidade da detecção precoce das alterações auditivas e estimular o desenvolvimento da autonomia da família sobre os cuidados para com sua saúde.

É necessário, portanto, que a educação e a informação sejam bases de um planejamento estratégico que objetivenão somentetranscender a resistência dos indivíduos, mas incentivar a participação ativa dos usuários nas ações de saúde com medidas que permitam maior alcance populacional possível dos programas.

De outro lado, é necessário o aprimoramento ou a reconstrução da relação profissional-usuário, prerrogativa indispensável no processo de efetivação da garantia dos direitos estabelecidos eassegurados pelo SistemaÚ nico de Saúde (SUS) e imprescindível para a conquista de maior autonomia dos usuários no cuidado da própria saúde.

\section{Colaboradores}

JC Fernandestrabalhou na concepção, desenvolvimento da pesquisa em todas as suas etapas, correção e redação final e M R N ozawa orientou a concepção, desenvolvimento da pesquisa e redação final. 
Referências

1. Santiago MR, Bassetto MCA. Triagem Auditiva em berçários. In: Bassetto M CA, Brock R, Wainsztejn R. Fonoaudiologia e Berçário: um convite à atuação. São Bernardo do Campo: Centro de Estudos Neomater; 1995. p. 61-64.

2. Oliveira TMT, Casarin MT, Souza MA, Marquett SCE, Barros TN. Atitudes dos M édicos Pediatras em relação à Audição Infantil. Pediatria Atual 1998;11(9):48-53.

3. Pagnossin DF, Oliveira TMT. Comparação das informações que pais de diferentes classes sociais têm sobre audição. Pró-Fono Rev. de Atual. Cient. 1998;10(1):8-16.

4. Soares E, Guerrero SM A, Azevedo M F. Estudo comparativo das triagens auditivas por emissões otoacústicas evocadas transientes, observação comportamental e medidas de imitância acústica em crianças com e se risco para deficiência auditiva. Revista Brasileira de Otorrinolaringologia 1998; 64(3):221-227.

5. Lima MCMP, Marba ST, Rossi TRF, Françozo M FC, Silva ABP, Lima GM L. Detecção de Alterações Auditivas em $\mathrm{N}$ eonatos [projeto de pesquisa]. Campinas: Universidade Estadual de Campinas; 2001.

6. Instituto Brasileiro de Geografia e Estatística. Censo Demográfico: dados nacionais sobre deficiência. Rio de Janeiro: IBGE; 2000.

7. Comitê Brasileiro Sobre Perdas Auditivas na Infância. Período Neonatal. Recomendação 01/99. Jornal do Conselho Federal de Fonoaudiologia 2000; (5):3-7.

8. National Infant Hearing. Consensus Statement (NIH). Early Identification of Hearing Impairment in Infants and Young Children. United States: NIH; 1993. p. 1-24.

9. Durante AS, Carvalho RMM, Costa MTZ, Cianciarullo MA, Voegels RL, Takahashi GM, Soares AVN, Spir EG. Programa de Triagem Auditiva Neonatal: modelo de implementação. Arq. Otorrinolaringol. 2004b; 8(1):56-62.

10. Joint Committee on Infant Hearing. [site on the Internet]. [cited 2004 Oct 04]. Available from: http:/ /www.jcih.org/default.htm

11. Yoshinaga-Itano C, Sedey AL, Coulter DK, Mehl $A L$. Language of early-and later-identified children with hearing loss. American Academy of Pediatrics 1998; 102(5):1161-1171.

12. Yoshinaga-Itano C. From screening to early identification and intervention: discovering predictors to successful outcomes for children with significant hearing loss. Journal of Deaf Studies and Deaf Education 2003; 8(1):11-30.

13. Françozo M FCF, Lima M CMP, Rossi TRF, Fernandes JC, Quinzan ER. Otoemissões: perfil dos pais de bebês que retornaram para a testagem. In: Anais do V Encontro de Pesquisa na Área de Serviço Social da PU C-Campinas e U nicamp; 2003; Campinas.

14. Santos $A A L$, Fernandes JR, Pinto LRCF. Incidência dos fatores de risco para deficiência auditiva em neonatos do programa de triagem auditiva da Prefeitura Municipal de Itanhaém/SP. In: Anais do II Congresso Brasileiro de Fonoaudiologia N eonatal. Jornada de Audiologia N eonatal; 2004; São Paulo.
15. Durante AS, Carvalho RMM , Costa MTZ, Cianciarullo M A, Voegels RL, Takahashi GM, et al. Programa de Triagem Auditiva Neonatal: modelo de implementação. Arq. Otorrinolaringol 2004; 8(1):56-62.

16. Rossi TRF, Françozo M FC, Tega LM, Fernandes JC, M endes LA. Triagem Auditiva neonatal: acompanhamento de bebês que falharam na primeira avaliação. In: Anais do I Congresso Brasileiro de Educação Especial. I Encontro da Associação Brasileira de Pesquisadores em Educação Especial. IX Ciclo de Estudos sobre Deficiência M ental. U niversidade Federal de São Carlos; 2003; São Carlos. p. 335-336.

17. Finitzo T, Albright $K, O^{\prime} N$ eal J. The Newborn with hearing loss: Detection in the nursery. Pediatrics 1998; 102(6):1452-1460.

18. Prieve B, Dalzell L, Berg A, Bradley M, Cacace A, Campbell D, DeCristofaro J, Gravel J, Greenberg E, Gross S, Orlando M, Pinheiro J, Regan J, Stevens F, Spivak L. New York State universal newborn hearing screening demonstration project: Inpatient outcome measures. Ear and Hearing 2000; 21(2):92-103.

19. Rouev P, Mumdzhiev H, Spiridonova J, Dimov P. Universal newborn hearing screening program in Bulgaria. Internet. J of Pediatric Otorhinolaryngology 2004; 68:805-810.

20. Chapchap MJ, Segre CAM. Programa de Triagem Auditiva N eonatal Universal e seus resultados. In: Anais do Congresso Internacional de Especialidades Pediátricas. M ódulo Reabilitação; 2000; Curitiba.

21. Triviños ANS. Introdução à pesquisa em Ciências Sociais: a pesquisa qualitativa em educação. $5^{a}$ ed. São Paulo: Atlas; 1995; 175-180.

22. Fleiss JL. Statistical M ethods for Rates and Proportions. $2^{\text {nd }}$ ed. New York: John Wiley \& Sons; 1981.

23. Conover WJ. Practical N onparametric Statistics. N ew York: John Wiley\& Sons; 1971.

24. Siegel S. Estatística não-paramétrica para as ciências do comportamento. São Paulo: M cGraw-Hill; 1975.

25. Hosmer DW, Lemeshow SL. Applied Logistic Regression. New York: John Wiley \& Sons; 1989.

26. Minayo MCS. 0 desafio do conhecimento: pesquisa qualitativa em saúde. 8a ed. São Paulo: Hucitec, 2004. p. 269.

27. Turato ER. Tratado da M etodologia da Pesquisa Qualitativa: construção teórico-epistemológica discussão comparada e aplicação nas áreas da saúde e humanos. Rio de Janeiro: Vozes; 2000.

28. Andersen RM. Revisiting the Behavioral Model and Access to M edical Care: Does It Matter? Journal of Health and Social Behavior 1995; 36(1):1-10.

29. Goddard M, Smith P. Equity of access to health care services: theory and evidence from the UK. Soc. Sci Med. 2001; 53:1149-1162.

30. Bosi M LM, Affonso KC. Cidadania, participação popular e saúde: com a palavra, os usuários da Rede Pública de Serviços. Cad Saude Publica 1998; 14(2):355-365.

Artigo apresentado em 26/05/2007

Aprovado em 13/12/2007

Versão final apresentada em 18/12/2007 